\title{
Correction of post-traumatic enophthalmos with anatomical absorbable implant and iliac bone graft
}

\author{
Ji Seon Choi, \\ Se Young Oh, \\ Hyung-Sup Shim \\ Department of Plastic and \\ Reconstructive Surgery, St. Vincent \\ Hospital, College of Medicine, The \\ Catholic University of Korea, Suwon, \\ Korea
}

\begin{abstract}
Background: Trauma is one of the most common causes of enophthalmos, and post-traumatic enophthalmos primarily results from an increased volume of the bony orbit. We achieved good long-term results by simultaneously using an anatomical absorbable implant and iliac bone graft to correct post-traumatic enophthalmos.

Methods: From January 2012 to December 2016, we performed operations on seven patients with post-traumatic enophthalmos. In all seven cases, reduction surgery for the initial trauma was performed at our hospital. Hertel exophthalmometry, clinical photography, three-dimensional computed tomography (3D-CT), and orbital volume measurements using software to calculate the specific volume captured on 3D-CT (ITK-SNAP, Insight Toolkit-SNAP) were performed preoperatively and postoperatively.

Results: Patients were evaluated based on exophthalmometry, clinical photographs, 3D-CT, and orbital volume measured by the ITK-SNAP program at 5 days and 1 year postoperatively, and all factors improved significantly compared with the preoperative baseline. Complications such as hematoma or extraocular muscle limitation were absent, and the corrected orbital volume was well maintained at the 1-year follow-up visit.

Conclusion: We present a method to correct enophthalmos by reconstructing the orbital wall using an anatomical absorbable implant and a simultaneous autologous iliac bone graft. All cases showed satisfactory results for enophthalmos correction. We suggest this method as a good option for the correction of post-traumatic enophthalmos.
\end{abstract}

Keywords: Absorbable implant / Autografts / Enophthalmos / Orbital wall fractures

\section{INTRODUCTION}

Enophthalmos is defined as posterior displacement of the eyeball due to volume changes in the bony orbit. It occurs when the balance between the bony orbit and the orbital contents (i.e., the globe, orbital fat, and muscular and ligamentous support) is disrupted [1]. Two to three millimeters of enophthalmos is

\footnotetext{
Correspondence: Hyung-Sup Shim

Department of Plastic and Reconstructive Surgery, St. Vincent Hospital, College of Medicine, The Catholic University of Korea, 93 Jungbu-daero, Paldal-gu, Suwon 16247 , Korea

E-mail: sharpshim@catholic.ac.kr

Received September 5, 2019 / Revised October 16, 2019 / Accepted October 17, 2019
}

clinically detectable, and more than $5 \mathrm{~mm}$ is disfiguring [2]. Enophthalmos can occur for a variety of reasons; enlargement of the orbital bony volume is a major cause, and another contributing factor is a decreased volume of orbital structures due to atrophy of the soft tissue in the orbit. Enophthalmos can be caused by congenital factors or by trauma, and post-traumatic enophthalmos accounts for the majority of cases. Furthermore, enophthalmos is the most common type of facial malformation that occurs after trauma, most likely as a sequela of facial fractures involving the orbit.

Many conventional strategies are currently used to correct post-traumatic enophthalmos. In addition to correction of the 
bony orbit, various other correction methods have been introduced, such as autogenous bone grafts, cartilage grafts, and the insertion of artificial prostheses (using absorbable or nonabsorbable materials). The choice of graft or implant material varies depending on the size and location of the defect, the curvature of the fractured orbit, the condition of the sinus, and the donor site preference of both the surgeon and the patient. Recently, various authors have proposed a calibration method using various artificial implant materials, such as polyethylene (Medpor), silicone-sheet implants, titanium mesh, metallic plates, and absorbable implants. In addition, several correction methods are available for autologous tissue, including bone grafts, diced cartilage grafts (nasal septal cartilage or costochondral cartilage), and fat injection [3-5]. However, each method has limitations, and controversy remains regarding which method is best.

We obtained good results in the correction of post-traumatic enophthalmos by simultaneously using an anatomical absorbable implant and an iliac bone graft. We propose this method as a useful approach for enophthalmos correction.

\section{METHODS}

The Internal Institutional Review Board of the Korea National Institute of Bioethics Policy approved this study. From January 2012 to December 2016, a total of seven patients with posttraumatic enophthalmos underwent surgery at a single center.
Patients who had a history of bony orbit trauma and an enlarged orbital cavity due to displacement of the orbital wall were included. To accurately measure the degree of improvement, only patients with unilateral injuries were included, with the exception of case 7 , in which the patient presented with bilateral eyeball injury after the initial trauma, but had a more severe injury on the left side. As a result, left enophthalmos developed. After the initial trauma, surgical repair of the right-side orbital wall fracture was performed. The right orbit was reconstructed to its normal anatomical morphology and we judged that it could be compared with the left orbit where enophthalmos occurred. Patients with a history of an operation to correct enophthalmos at other hospitals after the trauma and patients with underlying diseases that could affect orbital volume, such as thyroid disease, were excluded.

There were five male and two female patients, with a mean age of 39.7 years. The mean follow-up period was 1 year and 4 months. In five cases, the initial trauma was accompanied by trauma of other facial bones in addition to fracture of the orbital bone. Two cases had only an orbital bone fracture. There were four cases of multiple orbital bone fractures, and three cases had an orbital bone fracture on only one side. Reduction surgery for the initial trauma was performed at our hospital in all cases (Tables 1,2).

In all patients, Hertel exophthalmometry and three-dimensional computed tomography (3D-CT) were performed preoperatively, 5 days postoperatively, and 1 year postoperatively.

Table 1. Patient characteristics

\begin{tabular}{|c|c|c|c|c|c|c|}
\hline Patient no. & Age (yr) & Sex & Mechanism of injury & Associated injury site & Initial injured orbital wall & Initial surgery \\
\hline 1 & 18 & M & Motorcycle TA & $\begin{array}{l}\text { Left anterior and posterior table of } \\
\text { frontal sinus } \\
\text { Left zygoma } \\
\text { Left maxilla } \\
\text { Left mandible nasal bone }\end{array}$ & $\begin{array}{l}\text { Left medial wall \& floor } \\
\text { Left orbital roof } \\
\text { Left lateral wall }\end{array}$ & $\begin{array}{l}\text { Open reduction and internal fixation with } \\
\text { metallic plates and screws } \\
\text { Open reduction with absorbable mesh plate } \\
\text { insertion }\end{array}$ \\
\hline 2 & 41 & M & $\begin{array}{l}\text { Injury by fallen laminated } \\
\text { wood }\end{array}$ & $\begin{array}{l}\text { Left anterior and posterior table of } \\
\text { frontal sinus }\end{array}$ & $\begin{array}{l}\text { Left medial wall } \\
\text { Left orbital roof }\end{array}$ & $\begin{array}{l}\text { Open reduction with absorbable mesh plate } \\
\text { insertion }\end{array}$ \\
\hline 3 & 47 & M & $\begin{array}{l}\text { Kicking in the middle of } \\
\text { Taekwondo }\end{array}$ & NS & Right medial wall \& floor & $\begin{array}{l}\text { Open reduction with absorbable mesh plate } \\
\text { insertion }\end{array}$ \\
\hline 4 & 52 & $\mathrm{~F}$ & Fist injury & NS & Left floor & Open reduction with Medpor insertion \\
\hline 5 & 45 & M & Hit by a baseball & Right infraorbital rim & Right floor & $\begin{array}{l}\text { Open reduction and internal fixation with } \\
\text { absorbable plates and screws } \\
\text { Open reduction with Synpor insertion }\end{array}$ \\
\hline 6 & 56 & $\mathrm{~F}$ & Pedestrian TA & Right zygoma & Right medial wall \& floor & $\begin{array}{l}\text { Open reduction and internal fixation with } \\
\text { absorbable plates and screws } \\
\text { Open reduction with absorbable mesh plate } \\
\text { insertion }\end{array}$ \\
\hline 7 & 19 & M & Motorcycle TA & $\begin{array}{l}\text { Left zygoma } \\
\text { Right infraorbital rim } \\
\text { Right orbital bone }\end{array}$ & Both floor & $\begin{array}{l}\text { Open reduction and internal fixation with } \\
\text { absorbable plates and screws } \\
\text { Open reduction with absorbable mesh plate } \\
\text { and artificial bone graft }\end{array}$ \\
\hline
\end{tabular}

M, male; F, female; TA, traffic accident; NS, not significant. 
Table 2. Patient characteristics

\begin{tabular}{lcrl}
\hline Patient no. & $\begin{array}{c}\text { Interval between } \\
\text { injury and initial } \\
\text { reduction surgery }\end{array}$ & $\begin{array}{c}\text { Interval between initial } \\
\text { surgery and enophthalmos } \\
\text { correction surgery }\end{array}$ & Follow-up period \\
\hline 1 & $1.5 \mathrm{wk}$ & $10.5 \mathrm{mo}$ & $1 \mathrm{yr}$ \\
\hline 2 & $1.5 \mathrm{wk}$ & $9 \mathrm{mo}$ & $1 \mathrm{yr} 3 \mathrm{mo}$ \\
3 & $6 \mathrm{mo}$ & $9 \mathrm{mo}$ & $1 \mathrm{yr}$ \\
4 & $2 \mathrm{wk}$ & $11 \mathrm{yr} 11 \mathrm{mo}$ & $1 \mathrm{yr} 6 \mathrm{mo}$ \\
5 & $1.5 \mathrm{wk}$ & $11 \mathrm{mo}$ & $1 \mathrm{yr} 6 \mathrm{mo}$ \\
6 & $1.5 \mathrm{wk}$ & $1 \mathrm{yr} 4 \mathrm{mo}$ & $1 \mathrm{yr} 6 \mathrm{mo}$ \\
7 & $1.5 \mathrm{wk}$ & $9 \mathrm{mo}$ & $2 \mathrm{yr}$ \\
\hline
\end{tabular}

Clinical photography was performed before and after surgery. We compared the maximal degree of orbital wall depression on preoperative and 1-year postoperative 3D-CT compared with the healthy side (Fig. 1). The orbital volume was measured using the Insight Toolkit-SNAP (ITK-SNAP) software version 3.8 (Paul Yushkevich, Ph.D., University of Pennsylvania and Guido Gerig, Ph.D., University of Utah, USA) before the operation and at 1 year postoperatively. ITK-SNAP was used to analyze axial CT images by measuring the orbital area in each slice. In the program, the $3 \mathrm{D}$ morphology of the orbit is created using axial, coronal, and sagittal images. The total orbital area in each axial slice was bounded on each side by the medial and lateral orbital walls. Anteriorly, a line was drawn between the inner aspect of the orbital rim on each side. In the slices at the level of the medial canthus, a line was drawn between the lateral orbital rim and the anterior lacrimal crest. Posteriorly, a line was drawn across the openings of the orbital fissures and the optic canal (Supplementary Fig. 1).

The operation was performed under general endotracheal anesthesia and the incision was planned according to the part of the orbital wall that was most extensively involved. If the floor was the site of primary involvement, we approached the periosteum of the orbital rim using a subciliary incision. The depressed orbital floor was explored, the periosteum was elevated using a periosteal elevator, and the involved orbital wall was then completely exposed to subperiosteal dissection. At the same time, iliac bone was harvested en bloc, and the harvested amount was estimated using preoperative 3D-CT. A linear incision $(6-8 \mathrm{~cm})$ was made along the iliac crest from $2 \mathrm{~cm}$ posterior to the anterior superior iliac spine for the iliac bone graft. After releasing the abdominal musculature, the anterior iliac crest was exposed, with careful attention to prevent lateral femoral cutaneous nerve and vessel injury. Cancellous bone and corticocancellous strips were harvested in an en bloc manner, including the bone marrow, using an electrical saw and manual osteotome. The defects varied in size, with an average size of $3.0 \times 3.0 \times 1.5 \mathrm{~cm}$. The external contour of the ilium remained

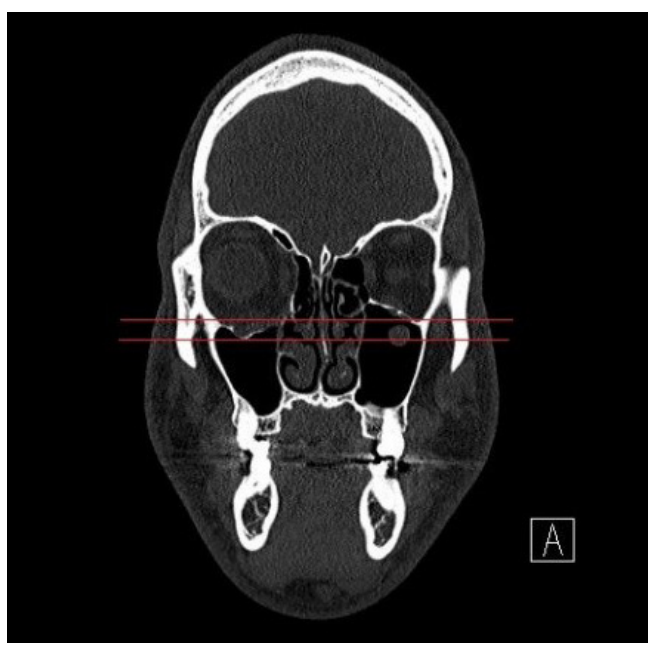

Fig. 1. Evaluation of the maximal depression point of the orbital wall on a coronal section of computed tomography compared with the contralateral side.

unaffected. The harvested bone was carved according to the $3 \mathrm{D}$ morphology of the defects as shown on preoperative 3D-CT. The estimated initial anatomical morphology of the affected orbit was modeled based on the patient's uninjured side, and the resultant hypothetical size of the bone graft was set as the size for calculating the bony defect in the axial, coronal, and sagittal planes on preoperative CT. After cutting the iliac bone to a size slightly larger than the measured defect size, the inner lining of the bone graft was carved into a curved shape and delicately adjusted to the contour of the orbit, as checked in the operating room. The new bony orbital wall was constructed using iliac bone, and the inner lining of the orbital wall was made using an anatomical absorbable implant of PLLA/PGA copolymer, LactoSorb sheet (Lorenz/Biomet, Jacksonville, FL, USA). We trimmed the anatomical absorbable implant to match the shape of the defect area based on the preoperative 3D-CT scan. First, the carved iliac bone graft was inserted into the defect area. The anatomical implant was then inserted between the bone graft and the ocular contents to create the inner lining of the orbital wall. The slightly empty area between the implant and the bone graft was filled with bone marrow particles obtained while harvesting the iliac bone graft. To prevent displacement of the inserted plate, fixation was performed using absorbable screws on the infraorbital rim. The incision was then closed and a suction drain was inserted if necessary. If required, a suction drain was also inserted at the iliac bone graft site, which was closed layer by layer (Fig. 2).

\section{RESULTS}

Exophthalmometry, clinical photographs, and 3D-CT were 



Fig. 2. Operative procedure. (A) The orbital rim was exposed through a subciliary incision. The involved orbital wall was completely exposed for subperiosteal dissection. A carved iliac bone graft and an anatomical absorbable implant were inserted into the defect area. The anatomical implant was inserted between the bone graft and the ocular contents to create the inner lining of the orbital wall. We fixed the anatomical absorbable implant onto the infraorbital rim using absorbable screws (arrow). (B) The iliac bone graft and anatomical absorbable implant were trimmed to be slightly larger than the defect. The iliac bone graft was further carved and attached to the undersurface of anatomical absorbable plate to just fit the area of the bone defect. (C) The iliac bone graft was attached to the undersurface of the anatomical absorbable plate.

taken routinely before the operation and at 5 days and 1 year after operation. The ITK-SNAP program was used to calculate the orbital volume preoperatively and 1 year postoperatively, and the preoperative and postoperative results were compared. The mean preoperative difference in exophthalmometry was 4 $\mathrm{mm}$, which improved to a mean of $1.57 \mathrm{~mm}$ at 5 days postoperatively. At 1 year, postoperatively, the mean difference in exophthalmometry was $1.71 \mathrm{~mm}$, indicating that the postoperative results remained stable compared with results at 5 days postoperatively. The mean preoperative orbital volume measured using ITK-SNAP was $37.56 \mathrm{~cm}^{3}$ and the mean postoperative orbital volume at 1 year postoperatively was $32.56 \mathrm{~cm}^{3}$. The rate of orbital volume on injury side compared to normal side decreased by $16.24 \%$. All data showed statistically significant differences. A comparison of the preoperative and postoperative clinical photographs showed that all patients obtained satisfactory esthetic results (Tables 3, 4).

Complications such as retrobulbar hematoma and limited eye movement were not observed. Bony reabsorption was not observed and the correction of enophthalmos was well maintained on 3D-CT scans at 1 year after the operation. There was no recurrence of enophthalmos. Two representative cases are presented below.

\section{Case 1 (patient no. 2)}

A 41-year-old man suffered a fracture of the left medial orbital wall, supraorbital rim, orbital roof, and anterior and posterior table of the frontal sinus from an injury inflicted by fallen laminate wood. Open reduction using an absorbable mesh plate was performed. After 8 months, the patient visited our hospital for the correction of post-traumatic enophthalmos. A 3D-CT evaluation revealed left-sided enophthalmos $(6 \mathrm{~mm}$ lower than the normal side). Surgery was performed through a subciliary incision. Iliac bone was harvested $(3.0 \times 2.5 \times 0.6 \mathrm{~cm})$ and carved into an anatomically suitable shape $(2.0 \times 1.0 \times 0.6 \mathrm{~cm})$ to fit the defect size. Postoperative complications were not observed. The patient was discharged at 10 days postoperatively without specific complications. The preoperative difference on exophthalmometry was $6 \mathrm{~mm}$, which improved to $3 \mathrm{~mm}$ postoperatively. The correction of enophthalmos and the patient's esthetic appearance were also satisfactory (Figs. 3, 4).

\section{Case 2 (patient no. 5)}

A 45-year-old man suffered a right orbital floor and infraorbital rim fracture due to being hit by a baseball. At 1.5 weeks after the injury, the orbital fractures were repaired with Synpor and open reduction using absorbable plates and screws. After 11 
Table 3. Measurement of preoperative and postoperative exo-ophthalmometer and maximal depression

\begin{tabular}{|c|c|c|c|c|c|c|c|}
\hline Patient no. & Age (yr) & Sex & $\begin{array}{l}\text { Difference of } \\
\text { exo-ophthalmometer/ } \\
\text { pre-op (mm) }\end{array}$ & $\begin{array}{c}\text { Difference of } \\
\text { exo-ophthalmometer/ } \\
\text { post-op } 5 \text { day }(\mathrm{mm})\end{array}$ & $\begin{array}{l}\text { Difference of } \\
\text { exo-ophthalmometer/ } \\
\text { post-op } 1 \text { yr (mm) }\end{array}$ & $\begin{array}{l}\text { Maximal depression } \\
\text { degree of orbital wall on } \\
\text { 3D CT/pre-op (mm) }\end{array}$ & $\begin{array}{c}\text { Maximal depression } \\
\text { degree of orbital wall on } \\
\text { 3D CT/post-op } 1 \text { yr (mm) }\end{array}$ \\
\hline 1 & 18 & M & 5 & 2 & 3 & 5 & 2 \\
\hline 2 & 41 & M & 6 & 3 & 3 & 7 & 2 \\
\hline 3 & 47 & $\mathrm{M}$ & 6 & 1 & 1 & 5 & 1 \\
\hline 4 & 52 & $\mathrm{~F}$ & 3 & 1 & 1 & 4 & 1 \\
\hline 5 & 45 & M & 5 & 3 & 3 & 5 & 2 \\
\hline 6 & 56 & $\mathrm{~F}$ & 2 & 1 & 1 & 3 & 1 \\
\hline 7 & 19 & $\mathrm{M}$ & 1 & 0 & 0 & 1 & 0 \\
\hline Mean & & & 4 & 1.57 & 1.71 & 4.28 & 1.28 \\
\hline
\end{tabular}

op, operative; 3D CT, three-dimensional computed tomography; M, male; F, female.

Table 4. Measurement of preoperative and postoperative orbital volume

\begin{tabular}{|c|c|c|c|c|c|}
\hline Patient no. & $\begin{array}{l}\text { Orbital volume: injury } \\
\text { side/normal side, } \\
\text { pre-op ( }\left(\mathrm{cm}^{3}\right)\end{array}$ & $\begin{array}{c}\text { Rate of orbital volume on injury } \\
\text { side compared to normal side, } \\
\text { pre-op (\%) }\end{array}$ & $\begin{array}{l}\text { Orbital volume: } \\
\text { injury side/normal side, } \\
\text { post-op } 1 \text { yr }\left(\mathrm{cm}^{3}\right)\end{array}$ & $\begin{array}{l}\text { Rate of orbital volume on injury } \\
\text { side compared to normal side, } \\
\text { post-op (\%) }\end{array}$ & $\begin{array}{c}\text { Difference of rate: } \\
\text { (rate at post-op)-(rate at pre-op) } \\
(\%)\end{array}$ \\
\hline 1 & $49.03 / 38.25$ & 128.13 & $40.24 / 37.85$ & 106.31 & -21.82 \\
\hline 2 & $46.45 / 36.74$ & 126.42 & $38.82 / 36.82$ & 105.43 & -20.99 \\
\hline 3 & $39.17 / 36.20$ & 120.52 & $35.69 / 36.15$ & 98.72 & -21.80 \\
\hline 4 & $39.15 / 32.50$ & 120.46 & $33.52 / 32.83$ & 102.13 & -18.33 \\
\hline 5 & $41.83 / 37.20$ & 112.44 & $36.95 / 36.90$ & 98.97 & -13.47 \\
\hline 6 & $23.18 / 20.24$ & 114.52 & $20.70 / 20.11$ & 102.93 & -11.59 \\
\hline 7 & $24.12 / 22.10$ & 110.45 & $22.01 / 21.01$ & 104.75 & -5.70 \\
\hline Mean & $37.56 / 31.89$ & 118.99 & $32.56 / 31.86$ & 102.74 & -16.24 \\
\hline
\end{tabular}

op, operative.

months, the patient wanted correction for post-traumatic enophthalmos. A 3D-CT evaluation revealed right-side enophthalmos ( $5 \mathrm{~mm}$ lower than the normal side). Surgery was performed through a subciliary incision. Iliac bone was harvested as needed $(3.2 \times 2.3 \times 1.0 \mathrm{~cm})$ and the harvested iliac bone was carved and trimmed to fit the defect size $(3.1 \times 2.0 \times 1.0 \mathrm{~cm})$. Postoperative complications were not observed. The patient was discharged 10 days after the operation without specific complications. The preoperative difference on exophthalmometry was $5 \mathrm{~mm}$, which improved to $3 \mathrm{~mm}$ postoperatively. The correction of enophthalmos and the patient's esthetic appearance were satisfactory (Figs. 5, 6).

\section{DISCUSSION}

Enophthalmos is caused by bony orbital volume enlargement due to bone fracture or orbital soft tissue atrophy. These factors can cause enophthalmos by contributing to posterior globe displacement [6]. Most cases of severe enophthalmos simultaneously involve both orbital volume enlargement due to a bone fracture and orbital soft tissue atrophy [7]. Surgical treatment of post-traumatic enophthalmos poses a high risk of damaging the optic nerve, orbit, and related structures during surgery; therefore, this treatment is considered to be technically difficult due to surgeons' fear regarding these risks. However, corrective surgery is necessary because enophthalmos often causes malformations, and if untreated, it may cause functional problems such as binocular diplopia. The correction of enophthalmos aims to restore the volume of the orbit and the position of its contents to improve both function and shape [1].

Various treatment methods for ocular depression have been reported. One method employs orbital wall reconstruction using osteotomy. Another option is to implant artificial materials or autologous tissue to replace the reduced orbital soft tissue. This approach can also be done by reducing the enlarged orbital volume by implanting artificial materials or autogenous bone or cartilage to replace the bony structure of the orbit.

Many surgeons consider enlargement of the orbital volume by displaced fractures as one of the major etiologies of enophthalmos and have corrected enophthalmos through bony structure reconstruction using osteotomy [7]. However, in enophthalmos caused by complicated facial bone trauma of the orbitozygomatic complex, not only is the orbital wall depressed, but the zygomatic bony structure itself is also distorted, which makes 



Fig. 3. Case 1 (patient no. 2), preoperative clinical photographs and computed tomography scan. (A, B) Preoperative clinical photographs, anteroposterior and worm's eye view. Evident hypoglobus and enophthalmos with sunken upper eyelid deformity were observed. (C, D) Preoperative computed tomography scan, coronal and axial section. The old orbital floor and medial wall fracture were observed on the left side.
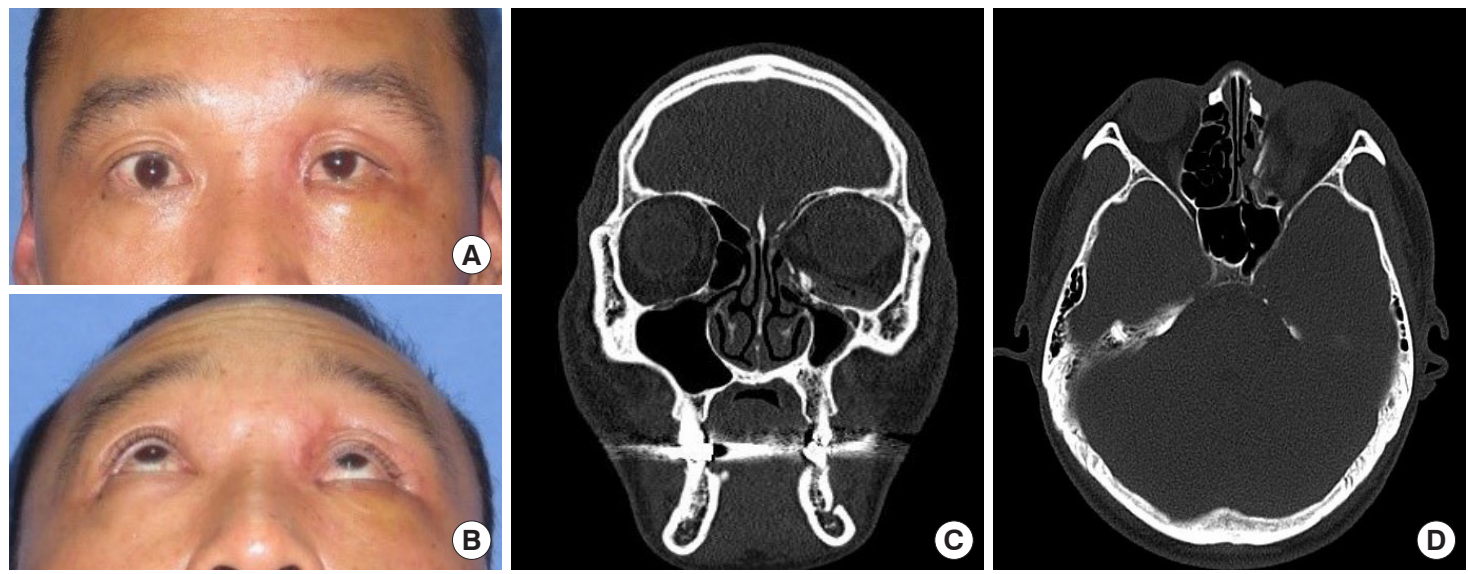

Fig. 4. Case 1 (patient no. 2), postoperative clinical photographs and computed tomography scan. (A, B) Postoperative clinical photographs, anteroposterior and worm's eye view. Fullness of the upper eyelid can be observed and enophthalmos improved significantly. (C, D) Postoperative computed tomography scan, coronal and axial section. The iliac bone graft was inserted into the orbital floor and medial wall. The orbital wall was reconstructed relatively symmetrically compared to the healthy orbital lining.
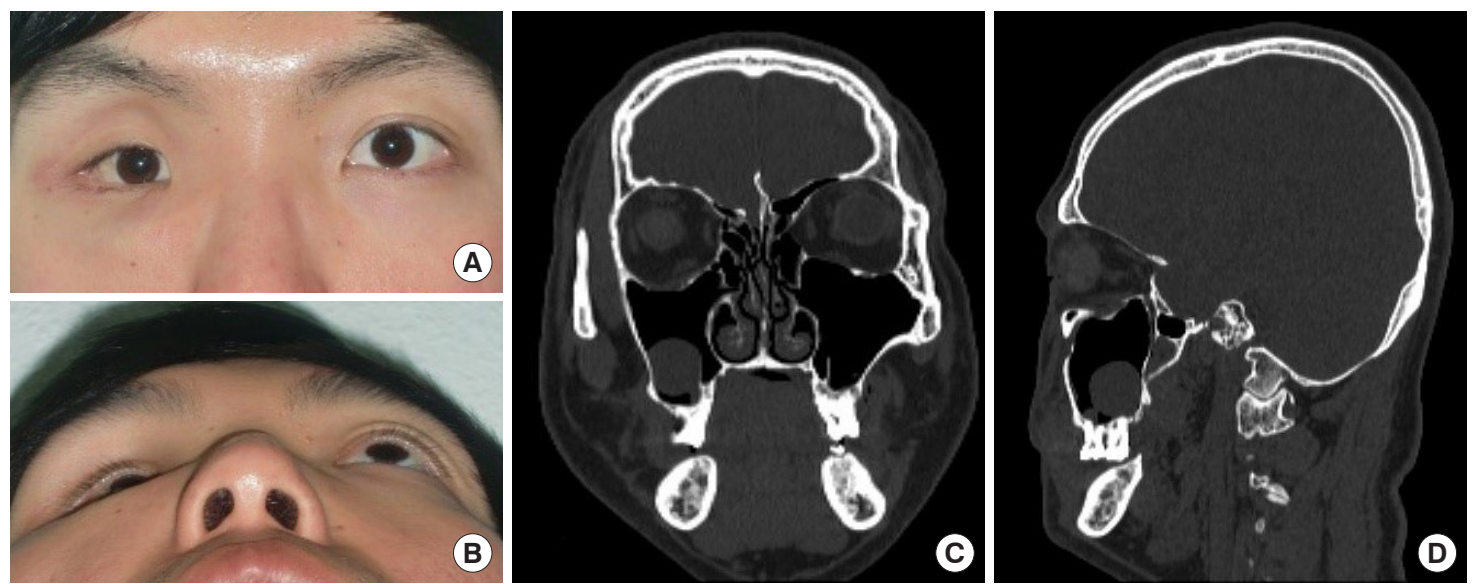

Fig. 5. Case 2 (patient no. 5), preoperative clinical photographs and computed tomography scan. (A, B) Preoperative clinical photographs, anteroposterior and worm's eye view. Hypoglobus and enophthalmos were evident on the left side. (C, D) Preoperative computed tomography scan, coronal and sagittal section. The old orbital floor fracture was evident. 

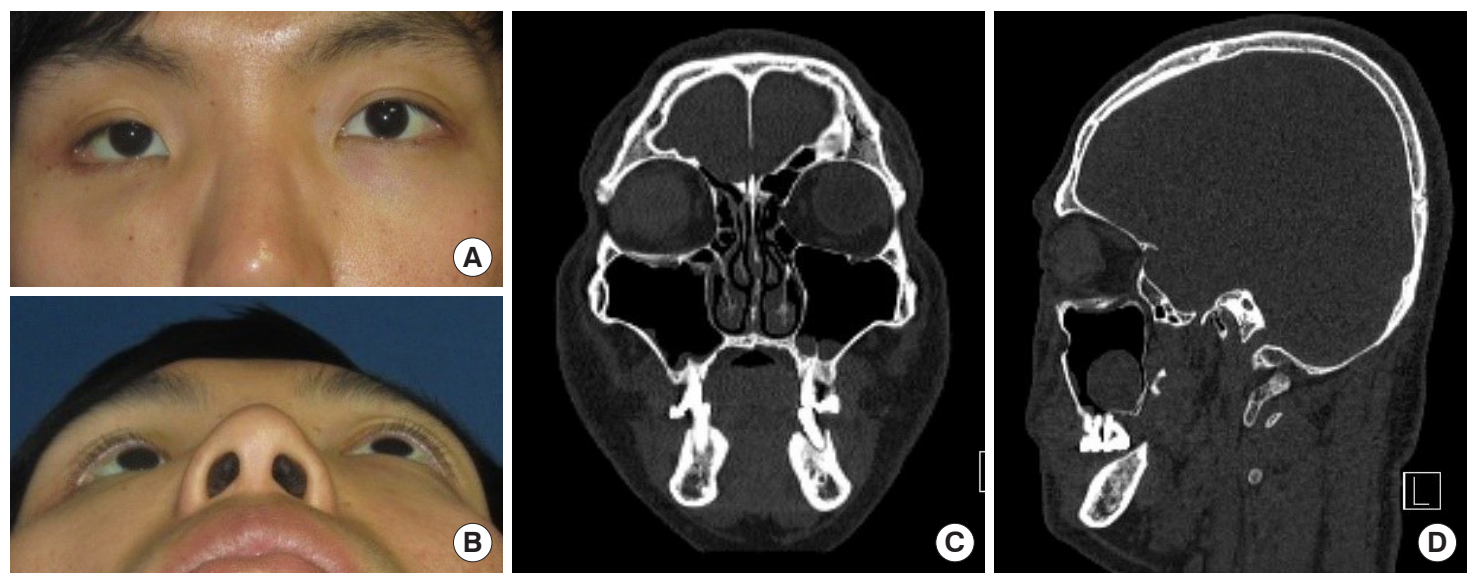

Fig. 6. Case 2 (patient no. 5), postoperative clinical photographs and computed tomography scan. (A, B) Postoperative clinical photographs, anteroposterior and worm's eye view. Enophthalmos and hypoglobus had improved significantly. (C, D) Postoperative computed tomography scan, coronal and sagittal section. The iliac bone graft was inserted into the orbital floor. Relatively good symmetry of the globe position was obtained.

the correction method much more complicated. In these cases, new osteotomy can be attempted on the posterior orbital wall. However, the interior orbit is a difficult anatomical area to approach and it can only be exposed to a limited degree. The risk of damaging the optic nerve and eye globe toward the posterior orbit makes it difficult to conduct a proper exploration. For these reasons, failure to reconstruct the original bone anatomy often occurs. Aside from it being practically difficult to achieve adequate anatomical bony reduction, another confounding factor is the actual cause of enophthalmos. Although bony volume expansion has been widely accepted as a major etiological cause for enophthalmos, it is not the only relevant causative factor. Many studies have shown that reduction of the orbital soft tissue is an important cause of enophthalmos. Repositioning of the surrounding bony segment by osteotomy can reduce the orbit volume, but completely correcting the posterior displacement of the orbit caused by scarring and atrophy of soft tissue in the orbit is difficult. Thus, volume manipulation should be considered as a major part of the treatment strategy.

Many surgeons have searched for the most appropriate surgical method for correcting enophthalmos in addition to correcting the anatomical bony structure with osteotomy. Various surgical methods have been reported, including the use of artificial alloplastic implants and autologous tissue. Correction methods using artificial alloplastic implants have been widely reported. Nonabsorbable materials have been used in these methods, such as polyethylene (Medpor), silicone-sheet implants, and titanium mesh. These are easy to adapt to complex orbital structures, can be readily shaped into the desired configurations, and have the advantage of providing sufficient volume for enophthalmos calibration alone. However, since they remain as permanent foreign bodies, several late-onset complications have been reported, such as infection, extrusion, implant migration, and residual diplopia [8]. Fortunately, we have not experienced any such complications.

To overcome the obstacles presented by these nonabsorbable materials, the use of absorbable materials has recently been reported. Absorbable materials include hydroxyapatite, poly-Llactide plates, poly-glycolide, and their composites. Absorbable plates are relatively soft, easy to manipulate, and show complete reabsorption, leaving no foreign material in the human body. However, because most absorbable materials are thin, they may not be sufficient to provide the volume needed for enophthalmos correction alone. After thorough resorption of the plate, the connective tissue around the absorbable plate is replaced by areas of fibrosis that may support the orbital structure. However, some studies have reported that the connective fibrotic tissue around the absorbable mesh plate cannot completely replace the original bony structures after resorption of the plate, mainly in the central portion of the defect. In these cases, enophthalmos may not be sufficiently corrected and may recur [9].

Notably, because most alloplastic implants are rigid, whether absorbable or nonabsorbable, fully adapting the shape of the implant to the anatomy of the orbit is difficult. Therefore, after insertion of the implant, there must be an empty space between the implant and the orbit. This is also a reason why the volume change of the orbit after implant insertion is not completely predictable [10].

Therefore, we presented a method of correcting enophthalmos by reconstructing the orbital wall using an alloplastic absorbable implant and autologous iliac bone graft simultaneously. We used the LactoSorb sheet (Biomet Microfixation System, resorbable PLLA/PGA copolymer) and an iliac bone graft.

Autologous grafts have been reported by other authors, using 
cartilage or fat in addition to bone. Hunter and Baker successfully corrected enophthalmos by performing a fat injection within the intramuscular cone. However, Hunter and Baker [3] reported that injecting nonpurified fat into the retrobulbar muscle cone yielded good results overall, but a high reabsorption rate. In many cases, recurrence has been reported after massive fat transplantation. Surgical methods using cartilage transplantation have also been reported, but cartilage is more flexible than bone, and it is difficult to support the orbital structures using cartilage.

In contrast, autologous bone has the following advantages: it can be harvested from various donor sites, such as calvarium, iliac bone, rib bone, and mandibular symphysis; it is hard enough to support the orbital structures; it is easily obtainable; and the resorption rate is also lower than that of cartilage or fat. Iliac bone has been used as a graft material in many types of surgery because it has a low donor site morbidity, can be used to harvest enough bone for enophthalmos correction, and results in a hidden surgical scar. The calvaria is also widely used as a donor site for harvesting large bone grafts. Compared with the calvaria, iliac bone is easier to obtain and, using a two-team surgical approach, can be performed simultaneously with enophthalmos correction surgery, saving operating time. In addition, the suitability of calvarial bone grafts remains controversial because of the possibility of neurological sequelae during the harvest process. Since the iliac bone is rough, it can injure the nerves or blood vessels of the orbit and cause discomfort during eyeball movement after surgery. Thus, an absorbable plate was inserted between the iliac bone graft and the orbital structure. We previously published a report on the reconstruction of large orbital floor defects using artificial bone substitutes and absorbable plates simultaneously. The postoperative discomfort and temporary diplopia incidence rate were very low because the absorbable plate helped to smooth the inner lining of the orbital wall. In addition, absorbable plates are considered appropriate for maintaining long-term orbital wall lining [11]. In the cases presented in that report, we used artificial bone substitutes, which provided sufficient volume to cover small defects such as orbital wall fractures, but would be insufficient to provide the volume needed for enophthalmos correction; furthermore, that technique would not be cost-effective. Since artificial bone is softer than autologous bone, it is likely to provide weaker support of the orbital structure. We experienced a case of enophthalmos after repair of an orbital wall fracture using artificial bone. However, no cases of recurrence were observed after this procedure with enophthalmos correction using autologous bone.

Since the iliac bone graft and absorbable plate are solid, an empty space is inevitably created between the iliac bone graft and absorbable plate. However, inserting the diced bone marrow chip into this space could ideally compensate this gap. Using an autologous bone graft and alloplastic absorbable plate at the same time, it was possible to overcome all the possible disadvantages of these materials when used separately, and to obtain all the advantages. Notably, one assumption may be that additional time is required to harvest the iliac bone. However, with an experienced surgeon and trained assistant, this delay can be sufficiently offset by the advantages of an autologous graft.

A limitation of this approach is that, like many autologous grafts, it is difficult to predict the survival and resorption rate of grafted bone. The survival rate of bone grafts is higher than that of other types of grafts, as the contact area with the recipient bone is large. In patients with post-traumatic enophthalmos, orbital bone is often lost during the injury, so insufficient contact with autologous bone is inevitable. Therefore, enophthalmos may recur during long-term follow-up due to absorption of graft tissue after surgery. Another limitation of this study is that it remained unknown whether enophthalmos recurred after 1 year because the follow-up results only extended up to 1 year. It is also difficult to carve bone in a way that precisely fits the defect size of the orbit wall. Therefore, it is challenging to obtain a perfect fit for the anatomy of the orbit. However, as an autologous graft, this strategy can be considered to have many advantages in terms of compensating for the disadvantages of artificial materials, such as foreign body infection, extrusion, implant migration, and residual diplopia. In addition, 3D-CT was used to minimize the possible errors that could occur before designing the graft.

There were no cases of complications such as recurrence of enophthalmos, hematoma, limitations of extraocular eye movement, and discomfort, even at the 1-year follow-up. All cases showed satisfactory results for enophthalmos correction. We suggest a surgical method using an anatomical absorbable implant and an iliac bone graft as a good option for the treatment of post-traumatic enophthalmos.

\section{NOTES}

\section{Conflict of interest}

No potential conflict of interest relevant to this article was reported.

\section{Ethical approval}

The study was approved by the Institutional Review Board of the Catholic University of Korea St. Vincent's Hospital (IRB No. 
VC19RESI0201) and performed in accordance with the principles of the Declaration of Helsinki. Written informed consents were obtained.

\section{Patient consent}

The patients provided written informed consent for the publication and the use of their images.

\section{ORCID}

Ji Seon Choi https://orcid.org/0000-0002-3531-0530

Se Young Oh https://orcid.org/0000-0001-9226-8386

Hyung-Sup Shim https://orcid.org/0000-0001-5156-2239

\section{Supplementary material}

Supplementary Fig. 1. ITK-snap (version 3.8 software) was used to analyze orbital axial computed tomography images by measuring the orbital volume on each slice.

Supplemental data can be found at:

https://doi.org/10.7181/acfs.2019.00451

\section{REFERENCES}

1. McRae M, Augustine HFM, Budning A, Antonyshyn O. Functional outcomes of late posttraumatic enophthalmos correction. Plast Reconstr Surg 2018;142:169e-178e.

2. Clauser L, Galie M, Pagliaro F, Tieghi R. Posttraumatic enophthalmos: etiology, principles of reconstruction, and correction. J Craniofac Surg 2008;19:351-9.

3. Hunter PD, Baker SS. The treatment of enophthalmos by orbital injection of fat autograft. Arch Otolaryngol Head Neck Surg 1994;120:835-9.
4. Metzler P, Ezaldein HH, Pfaff MJ, Parsaei Y, Steinbacher DM. Correction of severe enophthalmos by simultaneous fat grafting and anatomic orbital reconstruction. J Craniofac Surg 2014; 25:1829-32.

5. Pedemonte Trewhela C, Diaz Reiher M, Munoz Zavala T, Gonzalez Mora LE, Vargas Farren I. Correction of delayed traumatic enophthalmos using customized orbital implants. J Oral Maxillofac Surg 2018;76:1937-45.

6. Kim TH, Park IH, Hong SH, Eun SC. Sliced costochondral chip grafts in posttraumatic enophthalmos correction. J Craniofac Surg 2017;28:418-21.

7. Lee JW. Treatment of enophthalmos using corrective osteotomy with concomitant cartilage-graft implantation. J Plast Reconstr Aesthet Surg 2010;63:42-53.

8. Hwang K, Kim DH. Comparison of the supporting strength of a poly-L-lactic acid sheet and porous polyethylene (Medpor) for the reconstruction of orbital floor fractures. J Craniofac Surg 2010;21:847-53.

9. Baek WI, Kim HK, Kim WS, Bae TH. Comparison of absorbable mesh plate versus titanium-dynamic mesh plate in reconstruction of blow-out fracture: an analysis of long-term outcomes. Arch Plast Surg 2014;41:355-61.

10. Zhang Y, He Y, Zhang ZY, An JG. Evaluation of the application of computer-aided shape-adapted fabricated titanium mesh for mirroring-reconstructing orbital walls in cases of late posttraumatic enophthalmos. J Oral Maxillofac Surg 2010;68:20705.

11. Kwon H, Kim HJ, Seo BF, Jeong YJ, Jung SN, Shim HS. The role of resorbable plate and artificial bone substitute in reconstruction of large orbital floor defect. Biomed Res Int 2016;2016: 1358312. 